\title{
CARDIOMIOPATIA ARRITMOGÊNICA DO VENTRÍCULO DIREITO EM CÃES DA RAÇA BOXER: ATUALIDADES NO DIAGNÓSTICO E TRATAMENTO
}

\author{
(Arrhythmogenic right ventricular cardiomyopathy in the Boxer dog: updates on \\ diagnosis and treatment) \\ Elizabeth Regina Carvalho ${ }^{1 *}$, Michelli Fenerich ${ }^{1}$, Evandro Zacché ${ }^{1}$, Aparecido Antonio Camacho ${ }^{1}$, \\ Marlos Gonçalves Sousa ${ }^{2}$ \\ 1Universidade Estadual Paulista "Júlio de Mesquita Filho", Jaboticabal, São Paulo, Brasil. *Corresponding \\ author: beth rcarvalho@hotmail.com \\ ${ }^{2}$ Universidade Federal do Paraná, Curitiba, Paraná, Brasil.
}

RESUMO: A cardiomiopatia arritmogênica do ventrículo direito (CAVD) é uma doença miocárdica hereditária comumente observada no Boxer adulto, caracterizada pela substituição dos cardiomiócitos do ventrículo direito (VD) por tecido fibroadiposo, gerando áreas propensas à formação de arritmias ventriculares, que podem culminar em morte súbita. Devido aos avanços no mapeamento genético em cães domésticos, pode-se concluir que os Boxers são acometidos pela CAVD de maneira similar ao que ocorre em seres humanos, e, portanto, são considerados um modelo animal natural para o estudo da CAVD na espécie humana. Não há um teste de diagnóstico único e específico para CAVD, portanto, em Medicina Veterinária o diagnóstico baseia-se na presença de uma combinação de achados, que podem incluir presença de taquiarritmia ventricular sem outras causas documentáveis para a arritmia, síncope e histórico familiar de CAVD. O tratamento é direcionado à redução da frequência e complexidade da arritmia ventricular, e sotalol e/ou mexiletine são os antiarrítmicos mais comumente prescritos, bem como o ômega 3 advindo do óleo de peixe. Entretanto, não é sabido se o tratamento em cães assintomáticos está associado à melhores prognósticos. Embora alguns cães acometidos apresentem morte súbita ou desenvolvam insuficiência cardíaca congestiva, muitos deles têm arritmias ventriculares controláveis por antiarrítmicos e vivem uma vida normal. Algumas características dos complexos ventriculares prematuros, tais como polimorfismo e taquicardia ventricular, além da presença de sinais clínicos, e disfunção sistólica ventricular esquerda e direita estão associados à piores prognósticos.

Palavras-chave: arritmia; arritmogênese; eletrocardiografia; Holter; prognóstico

\begin{abstract}
The arrhythmogenic right ventricular cardiomyopathy (ARVC) is an inherited myocardial disease commonly observed in the adult Boxer, characterized by the replacement of right ventricle $(\mathrm{RV})$ cardiomyocytes by fibrofatty tissue, what generate areas prone to the formation of ventricular arrhythmias, which may culminate in sudden death. Due to advances in genetic mapping in domestic dogs, it could be concluded that Boxers are affected by ARVC in a closed related way to human beings, and therefore are considered a natural animal model for the study of human ARVC. There is not a single and specific diagnostic test for ARVC, thus, in veterinary practice the diagnosis is best based on a combination of findings, which may include the presence of ventricular tachyarrhythmia with no other documented causes for the arrhythmia, as well as syncope, and family history to ARCV. Treatment is directed to decrease the occurrence and the complexity of the ventricular arrhythmias, and sotalol and/or mexiletine are the antiarrhythmics most
\end{abstract}


commonly prescribed, as well as omega 3 from fish oil. However, it is unknown if the treatment on an asymptomatic dog prolongs survival. Although some affected dogs show sudden death or develop congestive heart failure, many of them have ventricular arrhythmias that are controllable by antiarrhythmics and live a normal life. Some characteristics of ventricular premature complexes, as polymorphism and ventricular tachycardia, the presence of clinical signs, and systolic dysfunction of left and right ventricles are associated with worst prognosis.

Keywords: arrhythmia; arrhythmogenesis; electrocardiography; Holter; prognosis

\section{INTRODUCTION}

A cardiomiopatia arritmogênica do ventrículo direito (CAVD) no Boxer é uma doença miocárdica familiar observada em adultos, caracterizada pela substituição dos cardiomiócitos do ventrículo direito (VD) por tecido fibroadiposo, gerando áreas propensas à formação de arritmias ventriculares (Meurs et al., 2014). Embora a CAVD tenha sido mais bem caracterizada no Boxer, casos isolados, suspeitos e confirmados, têm sido descritos no Dachshund (Simpson et al., 1994), Bullmastiff (Bright et al., 1995), Husky Siberiano (Palacio et al., 2001), Buldogue Inglês (Santilli et al., 2009; Nakao et al., 2011; Cunningham et al., 2018), Labrador Retriever (Nakao et al., 2011), Dálmata (Nakao et al., 2011), Pastor de Shetland (Nakao et al.,2011), e Weimaraner (Eason et al., 2015), além de ser descrita em felinos domésticos (Fox et al., 2000).

Indivíduos afetados pela CAVD são altamente predispostos à morte súbita, em alguns casos, como a primeira manifestação da doença (Corrado et al. 2017; Meurs et al., 2014). Pesquisas demonstram fortes semelhanças clínicas e patológicas da enfermidade em seres humanos e cães da raça Boxer, o que torna essa raça um modelo animal natural para o estudo da CAVD (Basso et al., 2004; Meurs et al., 2004).

A prevalência de CAVD em seres humanos é estimada em 1 caso a cada 5000 indivíduos na população em geral, e de 1 caso a cada 2000 pessoas em alguns países europeus, como a Itália e Alemanha (Rampazzo et al. 1994; Peters et al. 2004). A CAVD pode ser considerada a causa de até $10 \%$ das mortes súbitas cardíacas em pessoas com menos de 65 anos, e 22\% em atletas jovens (Kiès et al. 2006; Azaouagh et al. 2011). Embora a prevalência em Boxers não tenha sido estudada, clinicamente observa-se a CAVD como frequente causa mortis nessa raça, seja por insuficiência cardíaca congestiva, eutanásia ou morte súbita.

\section{ETIOLOGIA}

A CAVD, também conhecida como displasia arritmogênica do ventrículo direito, é uma doença miocárdica hereditária que afeta principalmente 0 ventrículo direito (VD) (Corrado et al. 2017). A perda do miocárdio ventricular direito com substituição dos cardiomiócitos por tecido fibroadiposo é a principal característica histopatológica desta doença (Thiene et al. 1988). Em seres humanos acometidos, infiltrados inflamatórios irregulares (principalmente linfócitos T) são frequentemente observados em associação com cardiomiócitos degenerados, sugerindo que o processo patológico pode ser imunologicamente mediado (Basso et al. 1996; Corrado et al. 1997).

A cicatriz fibroadiposa resultante estende-se do epicárdio até o endocárdio, e envolve predominantemente a parede livre do 
VD (Corrado et al. 1997). Em seres humanos, as lesões estão tipicamente localizadas na via de entrada do VD (região subtricuspídea), via de saída do VD (região infundibular), e ápice ventricular ("triângulo da displasia") (Marcus et al. 1982; Corrado et al. 1997).

Nos Boxers, etiologicamente, tratase de uma doença genética, de caráter autossômico dominante, com padrão de transmissão de penetrância incompleta (Oyama et al. 2008; Meurs et al. 2014). Mutações nos genes que codificam proteínas desmossômicas, especialmente no gene estriatina, localizado no cromossomo 17 (Meurs et al., 2010), importantes estruturas na adesão célula a célula, desempenham um papel fundamental na patogênese da CAVD (Corrado et al. 2017).

Seis loci independentes já foram identificados como possíveis causadores de mutações em quatro proteínas de ligação dos desmossomos: a desmoplaquina, placoglobina e placofilina, e estriatina (Indik et al., 2003; Macrae et al. 2006; Meurs et al. 2010). Além disso, ocorre mutação nos genes dos receptores cardíacos rianodina, o principal canal intracelular cardíaco de liberação de cálcio, tanto em cães da raça Boxer, quanto em seres humanos (Macrae et al. 2006; Oyama et al. 2008).

\section{INSTABILIDADE \\ ELETROFISIOLÓGICA}

A infiltração fibroadiposa no miocárdio apresenta instabilidade eletrofisiológica, predispondo ao desenvolvimento de circuitos elétricos reentrantes (Basso et al. 2009; Corrado et al. 2017). Assim, sabe-se que em seres humanos a estimulação adrenérgica pode aumentar a propensão às arritmias ventriculares ou até mesmo induzi-las diretamente (Corrado et al. 2017). A atividade esportiva aumenta 0 risco de morte súbita cardíaca em adolescentes e adultos jovens com CAVD (Thiene et al. 1988; Corrado et al. 2017). Sabe-se que em humanos afetados pela CAVD o exercício físico pode agravar o desacoplamento mecânico dos cardiomiócitos, desencadeando arritmias ventriculares malignas, e por esse motivo é considerado um fator ambiental crítico para o desenvolvimento e progressão da doença (Corrado et al. 2017).

A CAVD é uma causa importante de morte súbita cardíaca em humanos jovens e atletas (Corrado et al. 2017). Durante anos notou-se que os Boxers eram predispostos ao desenvolvimento de arritmias ventriculares e morte súbita (Basso et al. 2004). Devido aos avanços no mapeamento genético em cães domésticos, pode-se concluir que os Boxer são acometidos pela CAVD de maneira similar ao que ocorre em pessoas, e portanto, são considerados um modelo animal natural para o estudo da CAVD humana (Basso et al. 2004).

Em seres humanos, diversos marcadores eletrocardiográficos têm sido estudados como ferramenta diagnóstica e na estratificação de risco em indivíduos com doenças cardíacas. Dentre tais marcadores, destacam-se a dispersão do QRS, definida como a diferença máxima entre a duração do complexo QRS entre derivação precordial direita (rV2 nos animais) e esquerda (V4 nos animais) (Peters et al., 1999), a dispersão do intervalo QT, definida como a máxima diferença na duração do intervalo QT na eletrocardiografia de 12 derivações (Tse et al., 2016), o JTpico/JT, razão entre o JTpico (intervalo entre ponto $\mathrm{J}$, ou seja, término do complexo QRS, e pico da onda T) e JT (ponto $\mathrm{J}$ até o final da onda $\mathrm{T}$ ), avaliada em pequenos animais na derivação bipolar D II, e pré-cordiais rV2 e V4 (Moise et al., 1990; Coronel et al., 2009), ilustrados na Figura 1.

Figura 1 - Esquema representando a 
mensuração de marcadores eletrocardiográficos. A) Intervalo QT e subintervalos (QRS, JTpico e JT). B) Marcadores eletrocardiográficos utilizados na avaliação das arritmias ventriculares.

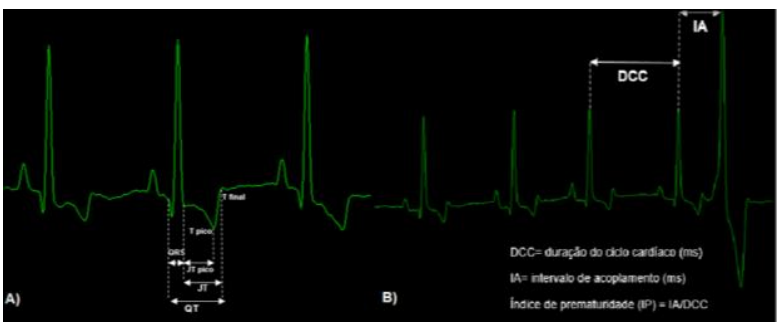

Enquanto a dispersão do QRS é um indicador da diferença na velocidade de condução do impulso elétrico entre duas regiões miocárdicas distintas, e, portanto, reflete a despolarização cardíaca, a dispersão do intervalo QT reflete a diferença no potencial de ação entre duas regiões do miocárdio, ou seja, é um marcador eletrocardiográfico de repolarização cardíaca, da mesma forma que o índice JTpico/JT. O intervalo de acoplamento (IA), definido como o intervalo R-R entre o batimento ectópico e o sinusal precedente, e o índice de prematuridade, razão entre o IA e o intervalo $R-R$ dos dois batimentos sinusais precedentes, são parâmetros eletrocardiográficos utilizados na classificação e estratificação de risco das arritmias ventriculares em seres humanos e animais (Igarashi et al., 2012; Kim et al., 2014; Carvalho et al., 2018). Em cães da raça Boxer, estudos que avaliem a utilidade de tais marcadores de arritmogênese aumentada são escassos.

\section{APRESENTAÇÃO CLÍNICA}

A CAVD no Boxer é mais comumente diagnosticada entre 5 e 7 anos de idade, embora alguns animais possam ser identificados precocemente, com 1 a 3 anos (Meurs, 2004; Meurs, 2017).

Nos Boxers, existem três formas clínicas da doença, propostas por Harpster et al (1991). Na categoria I ou forma oculta, os cães são assintomáticos e identifica-se pequeno número de arritmias ventriculares. $\mathrm{Na}$ categoria II ou forma evidente, os cães apresentam síncopes e cansaço fácil, principalmente durante exercícios ou momentos de excitação, associados à presença de extra-sístoles monomórficas. Na categoria III ou forma de disfunção do miocárdio, que é a menos comum, os animais apresentam sinais de insuficiência cardíaca congestiva, como tosse, intolerância ao exercício, efusões e síncope. Nesta fase, há presença de taquiarritmias ventriculares e ocasionalmente, taquiarritmias supraventriculares, como a fibrilação atrial (Harpster et al., 1991), além de disfunção sistólica biventricular, diagnosticada por meio de parâmetros ecocardiográficos de função sistólica radial (fração de encurtamento, strain e strain rate radiais e circunferenciais do ventrículo esquerdo, fractional area change (FAC) do ventrículo direito), e/ou função sistólica longitudinal (excursão sistólica do plano anular mitral ou tricúspide, MAPSE e TAPSE, respectivamente, strain e strain rate longitudinais) .

Ainda não está definido se as formas de manifestação da doença representam uma continuidade entre elas ou se são diferentes expressões de manifestações genéticas distintas (Harpster et al., 1991), embora saiba-se que os Boxers homozigotos para a mutação de deleção do gene estriatina são mais propensos a manifestação da categoria III da CAVD (Meurs et al., 2010). Alguns animais apresentam um grande número de eventos arrítmicos e não progridem para a forma sintomática da doença, enquanto outros, com o mesmo grau de ectopia ventricular progridem gradualmente e podem desenvolver sinais clínicos secundários às arritmias (Harpster et al., 1991; Meurs et al., 2014). 


\section{DIAGNÓSTICO}

Com o intuito de padronizar o diagnóstico da CAVD em humanos, em 1994 uma força-tarefa internacional propôs diretrizes sob a forma de um sistema de pontuação qualitativa com critérios maiores e menores (McKenna et al. 1994). Em 2010, as diretrizes foram revisadas para melhorar a sensibilidade diagnóstica, incluindo principalmente o rastreio de membros familiares dos acometidos, critérios quantitativos para o diagnóstico de anormalidades do ventrículo direito e adição de critérios genéticos moleculares (Marcus et al. 2010).

Em pessoas com suspeita de CAVD são avaliados: a função global e regional do VD, por meio da ecocardiografia bidimensional, ressonância magnética e angiografia; caracterização tecidual do VD na biópsia miocárdica; anormalidades eletrocardiográficas de repolarização, como a presença de onda $T$ negativa nas derivações pré-cordiais direitas; anormalidades eletrocardiográficas na condução e despolarização, como a presença de onda épsilon, episódios confirmados de taquicardia ventricular sustentada ou paroxística com morfologia de bloqueio de ramo esquerdo; e histórico familiar de CAVD em parentes de primeiro ou segundo grau. Para cada um desses tópicos, critérios maiores e menores são atribuídos.

Embora diversos aspectos sejam ponderados no diagnóstico da CAVD humana, existem situações nas quais podem haver equívocos no diagnóstico definitivo (Corrado et al. 2017). Taquicardia ventricular direita idiopática, sarcoidose cardíaca, doenças cardíacas congênitas que cursem em sobrecarga ventricular direita, e cardiomiopatia dilatada biventricular são alguns exemplos (Morin et al. 2010; Steckman et al. 2012; Corrado et al. 2017).
Em Boxers, o diagnóstico é melhor baseado na presença de uma combinação de fatores, incluindo histórico familiar da CAVD, presença de taquiarritmia ventricular, histórico de síncope ou intolerância ao exercício, e confirmação histopatológica post mortem de infiltração fibroadiposa no miocárdio do VD (Meurs, 2004).

\section{Exame físico}

Muitos cães acometidos pela CAVD não possuem anormalidades cardiovasculares detectáveis ao exame físico (Meurs, 2004; Meurs, 2017). A auscultação cardíaca deverá ser cuidadosa, uma vez que sopro geralmente não é auscultado em Boxers afetados pela CAVD, à exceção dos casos severos e menos comuns, nos quais há disfunção miocárdica (categoria III), e o sopro sistólico grau I a IV em ápice cardíaco esquerdo pode estar presente em $42 \%$ dos casos, consistente com regurgitação mitral secundária à dilatação ventricular (Meurs, 2004; Baumwart et al., 2005b; Meurs, 2017). Outras doenças cardíacas podem estar associadas ao mesmo padrão de sopro no Boxer, tais como estenose aórtica valvar ou subvalvar (Kvart et al., 1998; Koplitz et al., 2003), e devem portanto, serem excluídas. Ocasionalmente, pode ser detectada extrassístole ventricular prematura à auscultação, com déficit de pulso periférico (Meurs, 2004; Meurs, 2017). Pacientes na categoria III podem apresentar pulso jugular e ascite, atribuíveis à insuficiência cardíaca congestiva direita (Meurs, 2017).

\section{Eletrocardiografia}

Comumente, Boxers considerados afetados pela CAVD possuem registro eletrocardiográfico de 2-5 minutos sem arritmias ventriculares detectáveis, pois estas podem ser intermitentes e variáveis ao longo do tempo (Spier et al., 2004). Porém, quando detectados, 
complexos ventriculares prematuros (CVP) com morfologia de bloqueio de ramo esquerdo, avaliados em derivações planas frontais, como a aVF e D II (Kraus et al. 2002), conforme ilustrado pela Figura 2, podem estar presentes de forma isolada, em pares, trios, taquicardia ventricular ( $>3$ CVP em sequência) paroxística, ou ainda sustentada (> 30 segundos) (Spier et al., 2004; Meurs, 2004; Meurs, 2017).

Figura 2 - Traçado eletrocardiográfico de um cão da raça Boxer considerado acometido pela cardiomiopatia arritmogênica do ventrículo direito. Nota-se bigeminismo ventricular, e dois complexos ventriculares prematuros pareados após o terceiro batimento sinusal, seguido de pausa compensatória. A morfologia de bloqueio de ramo esquerdo nas derivações $D$ II e aVF sugerem a origem ventricular direita da arritmia.

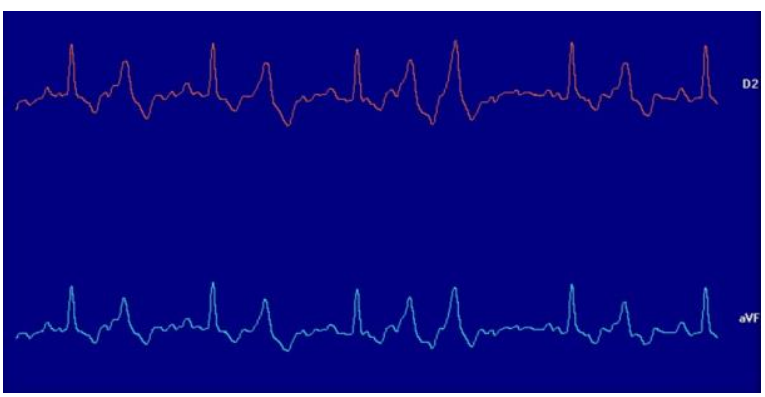

Eletrocardiografia ambulatorial (Holter)

A eletrocardiografia ambulatorial (Holter) é parte importante na triagem, diagnóstico e monitoramento da CAVD canina, na qual são investigados CVP (Meurs, 2004). Os CVP nos Boxers com CAVD são originados no VD e possuem morfologia de bloqueio de ramo esquerdo, quando avaliados no canal I (polo positivo em direção ao ápice do ventrículo esquerdo, similar à derivação D II).

Uma vez que a CAVD nos Boxers pode ser assintomática (categoria I ou forma oculta) em muitos animais acometidos, além do histórico familiar da doença nem sempre ser conhecido em cães, o diagnóstico clínico da CAVD muitas vezes passa a ser substanciado na quantidade e complexidade dos CVP no Holter de 24 horas, a despeito das inúmeras limitações dessa abordagem. As arritmias ventriculares podem ser originadas por outras doenças cardíacas que não a CAVD, como a degeneração mixomatosa da valva mitral (Crosara et al., 2010; Carvalho et al., 2018) e/ou distúrbios sistêmicos como a erliquiose (Ferreira et al., 2017) e neoplasias (Marino et al., 1994), e que, portanto, deverão ser excluídos com o auxílio de métodos diagnósticos complementares (p. ex. exames de sangue, ultrassonografia abdominal e ecocardiografia) antes de se estabelecer o diagnóstico de CAVD.

Atualmente não há consenso a despeito do número de CVP em 24 horas como ponto de corte para 0 diagnóstico da CAVD em Boxers. Alguns autores baseiam-se em guidelines não publicados do American College of Veterinary Internal Medicine Forum em 2009, e consideram que Boxers com mais de $300 \mathrm{CVP} / 24$ horas são fortemente suspeitos para a CAVD, enquanto entre 50 e $300 \mathrm{CVP} / 24$ horas a doença não pode ser descartada (Meurs et al., 2014; Meurs, 2017). Outros trabalhos sugerem que mais de 100 CVP/24 horas no Boxer adulto pode classifica-lo como provavelmente afetado, sobretudo se forem identificados padrões repetidos, como bigeminismo, trigeminismo ou taquicardia ventricular (Meurs, 2004). Cães adultos e saudáveis possuem em média 2 extrassístoles ventriculares prematuras em 24 horas (Meurs et al., 2001). Stern et al. (2010) realizaram um estudo com 301 Boxers, no qual os animais considerados clinicamente saudáveis apresentaram menos de 91 CVP isolados e monomórficos no Holter de 24 horas (Stern et al. 2010).

Ademais, pode haver variabilidade diária em relação ao número de CVP obtidos no Holter de 24 horas, e mudanças de cerca de $80 \%$ na 
frequência de arritmias ventriculares podem estar dentro do limite de variabilidade espontânea nos Boxers acometidos pela CAVD (Spier et al., 2004). Esta magnitude de variabilidade deverá ser considerada ao monitorar a resposta ao tratamento com antiarrítmicos, e também ao utilizar o Holter como ferramenta diagnóstica da CAVD no Boxer.

Eletrocardiografia de alta resolução (ECGAR)

O ECGAR é um método não invasivo que detecta a presença dos potenciais tardios (PT), ou seja, sinais de alta frequência e baixa amplitude que ocorrem ao final do complexo QRS (Calvert et al., 1998). Tais sinais não são detectados pela eletrocardiografia convencional, devido à baixa amplitude e interferência que os movimentos respiratórios e tremores musculares têm sob esta técnica (Calvert et al., 1998). Os PT são considerados marcadores da condução lenta, que ocorrem entre células lesadas do miocárdio após sofreram fibrose, fato que propicia o aparecimento de taquicardia ventricular por reentrada (Spier et al., 2004b). Portanto, a identificação dos PT pode ser utilizada como fator preditivo de risco para futuros eventos arrítmicos e morte súbita (Calvert et al. 1998; Moffa et al., 2001; Ferreira 2003; Barbosa et al., 2004; Bernardic et al., 2005; Spier et al., 2004b).

No Boxer com CAVD, a presença de PT correlaciona-se positivamente com morte súbita de origem cardíaca (Spier et al., 2001; Spier et al., 2004b). Porém, demonstrou-se a presença de PT em apenas $51,3 \%$ de cães Boxer com CAVD, e em $83,3 \%$ dos cães com CAVD na categoria III (Chamas, 2011), sugerindo que estes PT aparecem mais frequentemente nos estágios mais avançados da doença, à semelhança do que ocorre em seres humanos (Nava et al., 2000; Steriotis et al., 2009) e, portanto, não é considerado preditor precoce de mortalidade na CAVD do Boxer.

\section{Ecocardiografia}

Apesar da CAVD ser considerada uma doença miocárdica, o exame ecocardiográfico da maioria dos Boxers acometidos está dentro dos padrões de normalidade em relação à dimensão e função ventricular esquerda (Meurs, 2017). Como dito anteriormente, apenas uma minoria dos casos exibe disfunção miocárdica e dilatação ventricular. A sobrevida média nestes cães com dilatação ventricular esquerda é significativamente menor do que naqueles cujo o tamanho ventricular é considerado normal (Motskula et al., 2013). Um estudo avaliou o TAPSE como indicador da função sistólica ventricular direita e demonstrou que esta variável é menor nos Boxers com mais de $50 \mathrm{CVP} / 24 \mathrm{~h}$, e que o TAPSE reduzido é associado à doença mais severa e à piores prognósticos (Kaye et al., 2015).

\section{Exames de imagem}

A radiografia torácica geralmente encontra-se normal no Boxer com CAVD, exceto nos casos em que há dilatação ventricular ou biventricular, tornando-se passível de visibilizar cardiomegalia. Nos casos severos, evidências radiográficas de insuficiência cardíaca, tais como edema pulmonar e/ou efusão pleural, podem ser constatadas (Meurs, 2017).

A ressonância magnética cardíaca é um exame de imagem não invasivo que combina tanto a avaliação morfofuncional, quanto a caracterização tecidual, uma vez que a utilização de contraste possibilita a identificação de áreas miocárdicas fibrosadas (Perazzolo et al., 2015). Ainda pouco explorada em Medicina Veterinária para o diagnóstico da CAVD no Boxer, um estudo comparou a avaliação morfológica e 
funcional do ventrículo direito e esquerdo em Boxers afetados e controles saudáveis por meio da ressonância magnética cardíaca, e demonstrou prejuízos na função sistólica ventricular direita nos animais doentes (Baumwart et al., 2007).

\section{Biomarcadores}

A troponina cardíaca I mostrou-se significativamente elevada em alguns Boxers afetados pela CAVD, nos quais correlacionou-se com o número e complexidade da arritmia ventricular (Baumwart et al., 2007). Entretanto, há grande variabilidade nos níveis da troponina cardíaca I de caso para caso, e, portanto, a elevação nos seus níveis é um achado inconsistente nos cães acometidos pela CAVD (Meurs, 2017). A mensuração dos peptídeos natriuréticos cerebrais não mostrou ser um indicador útil da doença, a menos que ocorra disfunção miocárdica (Baumwart et al., 2005).

\section{Biopsia endomiocárdica}

Apesar de pouco difundida na rotina clínica veterinária, a biópsia endomiocárdica do VD por via transvenosa é o procedimento mais confiável para o diagnóstico preciso da CAVD em seres humanos, apesar da possibilidade de fornecer resultados falso negativos devido à natureza segmentar da doença (Basso et al., 1996). Além do caráter invasivo do procedimento, com risco de perfuração ventricular e tamponamento cardíaco, outra limitação da biópsia endomiocárdica é que, como ela é realizada por acesso intravenoso, a amostra obtida é de tecido subendocárdico, que pode não refletir o padrão transmural da infiltração fibroadiposa do miocárdio na CAVD (Turrini et al., 1999). Ainda, a biópsia endomiocárdica pode fornecer resultados duvidosos, uma vez que podem ser frequentemente encontradas, em indivíduos normais, ilhas de tecido adiposo entre os cardiomiócitos do VD (Mckenna et al., 1994; Thiene et al., 2001).

\section{Triagem}

Uma mutação genética de deleção no gene da estriatina (STRN), localizada no cromossomo 17, está associada ao desenvolvimento de CAVD em diversos cães da raça Boxer (Meurs et al., 2010), e, portanto, um kit comercialmente disponível

(http://www.laboklin.co.uk/laboklin/Genet icDiseases.jsp) tem sido utilizado para a triagem da doença em alguns países. A estriatina está localizada na região do disco intercalado nos cardiomiócitos, juntamente com outras três proteínas desmossômicas (placofilina-2, placoglobina, e desmoplaquina), todas envolvidas na patogênese da CAVD em seres humanos (Al-Jassar et al., 2013). $A$ deleção no STRN foi identificada em 94\% dos Boxers acometidos pela CAVD em um estudo, entretanto, também foi encontrada em 24\% dos controles saudáveis (Meurs et al., 2010). Embora a CAVD no Boxer seja conhecida como uma doença hereditária associada à deleção no STRN em muitos casos, é também uma doença familiar herdada com penetrância incompleta e expressão fenotípica variável (Meurs, 2017). Cães que são homozigotos para a deleção no STRN parecem apresentar uma forma mais grave de CAVD, demonstrada por um número maior de arritmias ventriculares, ocorrência de morte súbita e, em alguns casos, o desenvolvimento da categoria III da enfermidade (Meurs et al., 2010; Meurs et al., 2013).

\section{TRATAMENTO}

O tratamento da CAVD no Boxer geralmente é voltado para o controle das arritmias ventriculares, uma vez que 
a maior parte dos animais não desenvolve disfunção sistólica e não progride para insuficiência cardíaca (Meurs, 2017). Evidentemente, alguns cães assintomáticos afetados podem apresentar morte súbita como a primeira manifestação. Ainda não há um consenso a respeito do melhor momento para se iniciar a terapia antiarrítmica nos Boxers assintomáticos (Meurs, 2017).
No

características

ventriculares associadas ao desenvolvimento de sinais clínicos, como por exemplo: taquicardia ventricular sustentada, complexos ventriculares prematuros em padrões repetidos (ex. pares, trios, bigeminismo, trigeminismo), e fenômeno $\mathrm{R}$ em T (Meurs, 2004; Meurs, 2017) Porém, vale ressaltar que, no melhor do nosso conhecimento, não há estudos referentes ao impacto do tratamento com antiarrítmicos na sobrevida e prognóstico em cães da raça Boxer assintomáticos e acometidos pela CAVD . Com base nisso, alguns clínicos optam por iniciar a terapia uma vez que um determinado número de CVP no Holter de 24 horas tenha sido identificado ( $p$. ex. quando houver mais de 1000 CVP/24 h, ou se houver taquicardia ventricular ou quaisquer padrões repetidos de CVP) mesmo em um cão assintomático.

Foi demonstrado que nos Boxers, o tratamento antiarrítmico diminui tanto o número de CVP em 24 horas, quanto a complexidade da arritmia (MEURS et al., 2002), entretanto, a variabilidade diária no número de CVP registrados pelo Holter de 24 horas pode ser um fator de confundimento. Logo, ao optar pelo tratamento, os tutores deverão ser informados de que 0 uso de antiarrítmicos pode exercer um efeito contrário, pró-arrítmico (Meurs, 2017).

Cães acometidos pela CAVD e que apresentem síncope devem iniciar o tratamento antiarrítmico (Meurs, 2004; Meurs, 2017). Um estudo mostrou redução significativa dos episódios de síncope após a instituição da terapia (Meurs et al., 2002). As opções de antiarrítmicos que demonstraram reduzir o número e complexidade das arritmias ventriculares, bem como as síncopes, são o sotalol, na dose de 1,5 a $2 \mathrm{mg} / \mathrm{kg}$ via oral a cada 12 horas, e o mexiletine, na dose de 5 a $6 \mathrm{mg} / \mathrm{kg}$ via oral a cada 8 horas, sendo que este último não se encontra comercialmente disponível no Brasil. Ótimos resultados foram referidos com a associação de ambos (Meurs, 2017). A bradicardia neurocardiogênica é uma causa de síncope em cães da raça Boxer, embora menos comum que a CAVD, e não esteja obrigatoriamente relacionada à cardiomiopatia, embora possam coexistir (Thomason et al., 2008). Quando o Holter é realizado próximo ao evento da síncope no Boxer, e nenhum ou raros CVP são identificados, a síncope secundária à bradicardia deverá ser investigada (Thomason et al., 2008). O uso de betabloqueadores, ou de sotalol, pode agravar a bradicardia neurocardiogênica no Boxer (Thomason et al., 2008).

Idealmente, um controle pré e pós tratamento com Holter de 24 horas deve ser preconizado, para avaliar a resposta à terapia antiarrítmica, porém, animais sintomáticos e com arritmia ventricular observadas no ECG convencional deverão iniciar 0 tratamento o quanto antes (Meurs, 2017). Devido à variação diária no número de CVP documentados pelo Holter de 24 horas, preconiza-se que numa resposta positiva ao tratamento haja redução de cerca de $80 \%$ no número de CVP e na complexidade das arritmias (Meurs, 2017).

Ensaios clínicos em seres humanos demonstram efeitos cardioprotetores e antiarrítmicos dos ácidos graxos ômega 3 (Marchioli et al., 
1997; GISSI-Prevenzione Investigators, 1999; Albert et al., 2002). Em Boxers, o ômega 3 oriundo do óleo de peixe (ácido eicosapentanóico $78 \mathrm{mg}+$ ácido docosahexenóico $497 \mathrm{mg}$ ) administrado por 6 semanas consecutivas foi capaz de reduzir significativamente o número de CVP (Smith et al., 2007).

\section{PROGNÓSTICO}

Há sempre o risco de morte súbita em cães da raça Boxer acometidos pela CAVD. Entretanto, grande parte dos animais assintomáticos vivem por anos, mesmo sem tratamento (Meurs, 2017). Outra parcela, dos cães sintomáticos, podem ser manejados satisfatóriamente com antiarrítmicos durante anos (Meurs et al., 2014). Uma pequena parte destes, podem desenvolver disfunção sistólica e dilatação ventricular, nos quais a sobrevida é consideravelmente menor (Meurs et al., 2005; Meurs et al., 2014). Um estudo realizado com 122 Boxers, identificou que a presença de taquicardia ventricular, polimorfismo, presença de $>50 \mathrm{CVP} / 24 \mathrm{~h}$, idade acima de 4,6 anos, animais do sexo masculino e presença de dilatação ventricular esquerda na ecocardiografia são fatores preditores independentes de mortalidade cardíaca, tendo sido associados à piores prognósticos, a despeito da presença de sinais clínicos (Motskula et al., 2013). O TAPSE $<15,1$ $\mathrm{mm}$ está associado à menor sobrevida, mesmo nos Boxers sem disfunção sistólica (Kaye et al., 2015). Ademais, recentemente em uma investigação que incluiu 69 Boxers, foi demonstrado que a presença de síncope e episódios de taquicardia ventricular resultam em piores prognósticos (Chamas et al., 2016).

\section{REFERENCES}

ALBERT, C.M.; CAMPOS, H.; STAMPFER, M.J. et al. Blood levels of long-chain n-3 fatty acids and the risk of sudden death. New England Journal of Medicine, v.346, p.1113-1118, 2002.

AL-JASSAR, C.; BIKKER, H.; OVERDUIN, M. et al. Mechanistic basis of desmosome- targeted diseases. Journal of Molecular Biology, v.425, p.4006-4022, 2013.

AZAOUAGH, A.; CHURZIDSE, S.; KONORZA, T. Arrhythmogenic right ventricular cardiomyopathy/dysplasia: a review and update. Clinical Research in Cardiology, v. 100, p. 383-394, 2011.

BARBOSA, E. C.; BARBOSA, P. R. B.; BOMFIM, A. S. et al. Eletrocardiograma de alta resolução: a chave para a análise do método. Revista da Sociedade de Cardiologia do Estado do Rio de Janeiro, v. 17, n. 3, p. 201207, 2004.

BASSO, C.; THIENE, G.; CORRADO, D. et al. Arrhythmogenic right ventricular cardiomyopathy: dysplasia, dystrophy, or myocarditis? Circulation, v. 94, p. 983-991, 1996.

BASSO, C.; FOX, P.R.; MEURS, K.M. et al. Arrhythmogenic Right Ventricular Cardiomyopathy Causing Sudden Cardiac Death in Boxer Dogs A New Animal Model of Human Disease. Circulation, v.109, n.9, p. 1180-1186, 2004.

BASSO, C.; CORRADO, D.; MARCUS, F.I. et al. Arrhythmogenic right ventricular cardiomyopathy. Lancet, v. 373, p. 1289-1300, 2009.

BAUMWART, R.; MEURS, K.M. Assessment of plasma brain natriuretic peptide concentrations in Boxers with 
arrhythmogenic right ventricular cardiomyopathy. American Journal of Veterinary Research , v. 66, p. 20862089, 2005.

BAUMWART, R. D.; MEURS, K. M.; ATKINS, C. E. et al. Clinical, echocardiographic and electrocardiographic abnormalities in Boxers with cardiomyopathy and left ventricular systolic dysfunction: 48 cases. (1985-2003). Journal of the American Veterinary Medical Association, v. 226, n. 7, p. 1102-1104, 2005b.

BAUMWART, R.D.; ORVALHO, J.; MEURS, K.M. Evaluation of serum cardiac troponin I concentrations in Boxers with arrhythmogenic right ventricular cardiomyopathy. American Journal of Veterinary Research, v. 68, p. 524-528, 2007.

steckmanBERNADIC, M.; HUBKA, P.; SLAVKOVSKY, $P$. et al. High resolution electrocardiography in healthy dogs: time domain parameters and comparison of the non-stationary (Wigner distribution) versus standard stationary frequency domain analysis methods. Physiological Research, v. 54, n. 5, p. 477-484, 2005

BRIGHT, J. M.; McENTEE, M. Isolated right ventricular cardiomyopathy in a dog. Journal of the American Veterinary Medical Association, v. 207, n.1, p. 64-66, 1995.

CARVALHO, E.R.; AMPUERO, R.A.N.; TULESKI, G.L.R. et al. Polymorphism, coupling interval and prematurity index in dogs with degenerative mitral valve disease and ventricular arrhythmias. Veterinary Research Communications, mars (13), 2018.

CALVERT, C. A. High-resolution electrocardiography. The Veterinary
Clinics of North America - Small Animal Practice,v. 28, n. 6, p. 14291447, 1998.

CHAMAS, P. P. C. Estudo do eletrocardiograma ambulatorial, eletrocardiograma de alta resolução (ECGAR) e variabilidade da frequência cardíaca como indicadores prognósticos na cardiomiopatia arritmogênica de cães Boxer. 2011. São Paulo, 162 f. Tese (Doutorado em Ciências) - Faculdade de Medicina Veterinária e Zootecnia, Universidade de São Paulo.

CHAMAS, P.P.C.; OLIVEIRA, V.M.C.; YAMAKI, F.L. et al. Prognostic value of heart rate variability and Holter monitoring in Boxer dogs with arrhythmogenic right ventricular cardiomyopathy. Arquivo Brasileiro de Medicina Veterinária e Zootecnia, v.68, n.5, p.1219-1227, 2016.

CORONEL, R.; WILMS-SCHOPMAN, F.J.; OPTHOF, T. et al. Dispersion of repolarization and arrhythmogenesis. Heart Rhythm, v. 6, p. 537-543, 2009.

CORRADO, D.; BASSO, C.; THIENE, G. et al. Spectrum of clinicopathologic manifestations of arrhythmogenic right ventricular cardiomyopathy/dysplasia: a multicenter study. Journal of the American College of Cardiology, v. 30, p. 1512-1520, 1997.

CORRADO, D.; LINK, M. S.; CALKINS, $\mathrm{H}$. Arrhythmogenic Right Ventricular Cardiomyopathy. The New England Journal of Medicine, v. 376, p. 61-72, 2017.

CROSARA, S.; BORGARELLI, M.; PEREGO, M. et al. Holter monitoring in 36 dogs with myxomatous mitral valve disease. Australian Veterinary Journal, v. 88, p.386-392, 2010. 
CUNNINGHAM, S.M.; SWEENEY, J.T.; MacGREGOR, J. et al. Clinical features of english bulldogs with presumed arrhythmogenic right ventricular cardiomyopathy: 31 cases (2001-2013). Journal of the American Animal Hospital Association, v.54, n.2., p.95102, 2018.

EASON, B.D.; LEACH, S.B.; KUROKI, $\mathrm{K}$. Arrhythmogenic right ventricular cardiomyopathy in a Weimaraner. The Canadian Veterinary Journal, v.56, n.10, p.1035-1039, 2015.

FERREIRA, W. L. Estudo da eletrocardiografia de alta resolução em cães. 2003. Jaboticabal, 71 f. Tese (Doutorado em Clínica Médica Veterinária) - Faculdade de Ciências Agrárias e Veterinárias, Universidade Estadual Paulista (UNESP).

FERREIRA, G.B.; FILIPPI, M.G.; PAES, A.C. et al. Electrocardiographic evaluation in dogs with monocytic ehrlichiosis. Revista de Educação Continuada em Medicina Veterinária e Zootecnia do CRMV-SP, v.15, n.2, p.38-44, 2017.

FOX, P.R.; MARON, B.J.; BASSO, C. et al. Spontaneously occurring arrhythmogenic right ventricular cardiomyopathy in the domestic cat - a new animal model similar to the human disease. Circulation, v.102, n. 10, p. 1863-1870, 2000.

\section{GISSI-PREVENZIONE} INVESTIGATORS.

Dietary supplementation with n-3 polyunsaturated fatty acids and vitamin $E$ after myocardial infarction: Results of the GISSI-Prevenzione trial. LANCET, v.354, p.447-455, 1999.

GILMOUR, R. F. Restitution, heterogeneity and unidirectional conduction block: New roles for old players. Heart Rhythm, v. 6, p. 544$555,2009$.

HARPSTER, N. K. Boxer cardiomyopathy. In: KIRK, R.W. Current Veterinary Therapy VIII. Philadelphia : W.B. Saunders, 1983, p.329-337.

HARPSTER, N. K. Boxer cardiomyopathy. The Veterinary Clinics of North America Small Animal Practice, v. 21, p. 989-1004, 1991.

IGARASHI, M.; TADA H.; KUROSAKI, $\mathrm{K}$. et al. Electrocardiographic determinants of the polymorphic QRS morphology in idiopathic right ventricular outflow tract tachycardia. Journal of Cardiovascular Electrophysiology, v. 23, n. 5, p. 521-526, 2012.

INDIK, J. H.; MARCUS, F. I. Arrhythmogenic right ventricular cardiomyopathy/dysplasia. Indian pacing and electrophysiology journal, v. 3, p. 148-156, 2003.

KAYE, B.M.; BORGEAT, K.; MOTSKULA, P.F. et al. Association of tricuspid annular plane systolic excursion with survival time in Boxer dogs with ventricular arrhythmias. Journal of Veterinary Internal Medicine, v.29, p.582-588, 2015.

KOPLITZ, S.; MEURS, K.; SPIER, A. et al. Aortic ejection velocity in healthy boxers with soft murmurs and boxers without cardiac murmurs: 210 cases (1997-2001). Journal of American Veterinary Medical Association, v.222, p.770-774, 2003.

KVART, C.; FRENCH, A.T.; LUIS FUENTES, V. et al. Analysis of murmur intensity duration and frequency components in dogs with aortic stenosis. 
Journal of Small Animal Practice, v.39, p.318-324, 1998.

KIÈS, P.; BOOTSMA, M.; BAX, J. et al. Arrhythmogenic right ventricular dysplasia/cardiomyopathy: Screening, diagnosis, and treatment. Heart Rhythm, v. 3, p. 225-234, 2006.

KIM, Y. R; NAN, G.B.; KWON, C.H. et al. Second coupling interval of nonsustained ventricular tachycardia to distinguish malignant from benign outflow tract ventricular tachycardias. Heart Rhythm, v. 11, p. 2222-2230, 2014.

KNECHT, S.; SACHER, F.; WRIGHT, M. et al. Long-term follow-up of idiopathic ventricular fibrillation ablation a multicenter study. Journal of American College of Cardiology, v. 54, n. 6, p. 522-528, 2011.

KRAUS, M. S.; MOISE, N.S.; RISHNIW, $M$. et al. Morphology of ventricular arrhythmias in the boxer as measured by 12-lead electrocardiography with pace-mapping comparison. Journal of Veterinary Internal Medicine, v. 16, n. 2, p. 153-158, 2002.

KUROSAKI, K.; NOGAMI, A.; SHIRAI, Y. et al. Positive QRS complex in lead I as a malignant sign in right ventricular outflow tract tachycardia. Circulation, v. 77, n. 4, p. 968-974, 2013.

MACRAE, C. A.; BIRCHMEIER, W.; THIERFELDER, L. Arrhythmogenic right ventricular cardiomyopathy: moving toward mechanism. The journal of clinical investigation, v. 116, n. 7, p. 1825-1828, 2006.

MARCHIOLI, R.; BARZI, F.; BOMBA, E. et al. Early protection against sudden death by $n-3$ polyunsaturated fatty acids after myocardial infarction: Time-course analysis of the results of the Gruppo
Italiano per lo Studio della Sopravivenza nell'Infarto Miocardico (GISSI)Prevenzione. Circulation, v.105, p. 1897-1903, 2002.

MARCUS, F. I.; McKENNA, W.J.; SHERRILL, D. et al. Diagnosis of arrhythmogenic right ventricular cardiomyopathy/dysplasia: proposed modification of the Task Force Criteria. European Heart Journal, v. 31, p. 806814, 2010.

MARCUS, F. I.; FONTAINE, G.H.; GUIRAUDON, G. et al. Right ventricular dysplasia: a report of 24 adult cases. Circulation, v. 65, p. 384-398, 1982.

MARINO, D.J.; MATTHIESEN, D.T.; FOX, P.R. et al. Ventricular arrhythmias in dogs undergoing splenectomy: a prospective study. Veterinary Surgery, v.23, n.2, p.101-106, 1994.

MCKENNA, W. J.; THIENE, G.; NAVA, A. et al. Diagnosis of arrhythmogenic right ventricular dysplasia/cardiomyopathy. British heart journal, v. 71, p. 215-218, 1994.

MEURS, K. M.; SPIER, A.W.; WRIGHT, N.A. et al. Use of ambulatory electrocardiography for detection of ventricular premature complexes in healthy dogs. Journal of American Veterinary Medical Association, v. 218, n. 8, p. 1291-1292, 2001.

MEURS, K.M.; SPIER, A.W.; WRIGHT, N.A. et al. Comparison of the effects of four antiarrhythmic treatments for familial ventricular arrhythmias in Boxers. Journal of American Veterinary Medical Association, v. 221, p. 522-527, 2002.

MEURS, K. M. Boxer dog cardiomyopathy: An update. Veterinary Clinics of North America - Small Animal Practice, v. 34 , n. 5, p. $1235-$ 
1244, 2004.

MEURS, K.M.; MAUCELI, E.; LAHMERS, S. et al. Genome-wide association identifies a mutation in the 3 ' untranslated region of striatin, a desmosomal gene, in a canine Arrhythmogenic Right Ventricular Cardiomyopathy model of arrhythmogenic right ventricular cardiomyopathy. Human Genetics, v.20, p. 315-324, 2010.

MEURS, K. M.; STERN, J. A.; SISSON, $D$. D. et al. Association of dilated cardiomyopathy with the striatin mutation genotype in boxer dogs. Journal of Veterinary Internal Medicine, v.27, p.1437-1440, 2013.

MEURS, K. M.; STERN, J.A.; REINADORESTE, $Y$. et al. Natural history of arrhythmogenic right ventricular cardiomyopathy in the boxer dog: a prospective study. Journal of Veterinary Internal Medicine, v. 28, p. 1214-1220, 2014.

MEURS, K. M. Arrhythmogenic Right Ventricular Cardiomyopathy in the Boxer Dog: an update. Veterinary Clinics of North America - Small Animal Practice, v. 47, n. 5, p. 1103-1111, 2017.

MOFFA, P. J.; SANCHES, P. C. R. O eletrocardiograma de alta resolução. In: RAMIRES, J. A. F.; OLIVEIRA, S. A. Eletrocardiograma: normal e patológico. 7. ed. São Paulo: Editora Roca, 2001. p. 881-902.

MOISE, N. S.; GILMOR, J.R.; WALLEN, V. M. et al. Inherited ventricular ectopy and sudden death in young german shepherd dogs. Journal of the American College of Cardiology, v. 15, n. 2, p. 152, 1990.
MORIN, D. P.; MAUER, A.C.; GEAR, K. et al. Usefulness of precordial $\mathrm{T}$-wave inversion to distinguish arrhythmogenic right ventricular cardiomyopathy from idiopathic ventricular tachycardia arising from the right ventricular outflow tract. American Journal of Cardiology, v. 105, p. 1821-1824, 2010.

MOTSKULA, P.F.; LINNEY, C.; PALERMO, V. et al. Prognostic value of 24-hour ambulatory ECG (Holter) monitoring in Boxer dogs. Journal of Veterinary Internal Medicine, v.27, p.904-912, 2013.

NAKAO, S.; HIRAKAWA, A.; YAMAMOTO, S. et al. Pathological features of arrhythmogenic right ventricular cardiomyopathy in middleaged dogs. The Journal of Veterinary Medical Science, v.73, n.8, p.10311036, 2011.

NAVA, A.; FOLINO, A.F.; BAUCE, B. et al. Signal-averaged electrocardiogram in patients with arrhythmogenic right ventricular cardiomyopathy and ventricular arrhythmias. European Heart Journal, v. 21, n. 1, p. 58-65, 2000.

OYAMA, M. A.; REIKEN, S.; LEHNART, S. E. et al. Arrhythmogenic right ventricular cardiomyopathy in Boxer dogs is associated with calstabin2 deficiency. Journal of Veterinary Cardiology, v. 10, p. 1-10, 2008.

PALACIO, M.J.F.; BERNAL, L.J.; BAYON, A. et al. Arrhythmogenic right ventricular dysplasia/cardiomyopathy in a Siberian Husky. Journal of Small Animal Practice, v. 42, p. 137-142, 2001.

PERAZZOLO, M.M.; RIZZO, S.; BAUCE, B. et al. Arrhythmogenic right ventricular cardiomyopathy. Contribution 
of cardiac magnetic resonance imaging to the diagnosis. Herz, v.40, n.4, p.600606, 2015.

PETERS, S.; PETERS, H.; THIERFELDER, L. Risk stratification of sudden cardiac death and malignant ventricular arrhythmias in right ventricular dysplasia-cardiomyopathy. International Journal of Cardiology, v. 71, p. 243-250, 1999.

PETERS, S.; TRUMMEL, M.; MEYNERS, W. Prevalence of right ventricular dysplasia cardiomyopathy in a non-referral hospital. International Journal of Cardiology, v. 97, p. 499451, 2004.

RAMPAZZO, A.; NAVA, A.; DANIELI, G.A. et al. The gene for arrhythmogenic right ventricular cardiomyopathy maps to chromosome 14q23-q24. Human Molecular Genetics, v. 3, p. 959-962, 1994.

SANTILLI, R.A.; BONTEMPI, L.V.; PEREGO, $M$. et al. Outflow tract segmental arrhythmogenic right ventricular cardiomyopathy in an English Bulldog. Journal of Veterinary Cardiology, v. 11, p. 47-51, 2009.

SIMPSON, K. W.; BONAGURA, J. D.; EATON, K. A. Right ventricular cardiomyopathy in a dog. Journal of Veterinary Internal Medicine, v. 8, n.4, p. 306-309, 1994.

SPIER, A. W.; MEURS, K. M.; LINN, C. G. Signal-averaged ECG in the assessment of arrhythmogenic cardiomyopathy in Boxers. In: AMERICAN CONFERENCE OF VETERINARY INTERNAL MEDICINE, Denver, Abstracts 2001, p. 872.

SPIER, A.W.; MEURS, K.M.; LEHMKUHL, L.B. et al. Evaluation of spontaneous variability in the frequency of ventricular arrhythmias in Boxers with arrhythmogenic right ventricular cardiomyopathy. Journal of American Veterinary Medical Association, v. 224, p.538-541, 2004.

SPIER, A. W.; MEURS, K. M. Use of signal-averaged electrocardiography in the evaluation of arrhythmogenic right ventricular cardiomyopathy in Boxers. Journal of the American Veterinary Medical Association, v. 225, n. 7, p.1050-1055, 2004b.

SMITH, C.E.; FREEMAN, L.M.; RUSH, J.E. et al. Omega-3 fatty acids in boxer dogs with arrhythmogenic right ventricular cardiomyopathy. Journal of Veterinary Internal Medicine, v.21, p.265-273, 2007.

STECKMAN, D.A.; SCHNEIDER, P.M.; SCHULLER, J.L. et al. Utility of cardiac magnetic resonance imaging to differentiate cardiac sarcoidosis from arrhythmogenic right ventricular cardiomyopathy. American Journal of Cardiology, v. 110, p. 575-579, 2012.

STERIOTIS, A. K.; BAUCE, B.; DALIENTO, L. $\quad$ et al. Electrocardiographic pattern in arrhythmogenic right ventricular cardiomyopathy. The American Journal of Cardiology, v. 103, n. 9, p.1302-1308, 2009.

STERN, J.A.; MEURS, K.M.; SPIER, A.W. et al. Ambulatory electrocardiographic evaluation of clinically normal adult Boxers. Journal of the American Veterinary Medical Association, v. 236, n.4, p.1-4, 2010.

STETTLER, C.; BEARTH, A.; ALLEMANN, $S$. et al. QTC interval and resting heart rate as long-term predictors of mortality in type 1 and type 2 diabetes mellitus: a 23-year follow-up. Diabetologia, v. 50, p. 186-194, 2007. 
THIENE, G.; NAVA, A.; CORRADO, D. et al. Right ventricular cardiomyoathy and sudden death in young people. The New England Journal of Medicine, $v$. 318, n. 3, p. 129-133, 1988.

THIENE, G.; BASSO, C. Arrhythmogenic right ventricular cardiomyopathy: an update. Cardiovascular Pathology, v. 10, p. 109-117, 2001.

THOMASON, J.D.; KRAUS, M.S.; SURDYK, K.K. et al. Bradycardiaassociated syncope in 7 Boxers with ventricular tachycardia (2002-2005). Journal of Veterinary Internal Medicine, v. 22, n.4, p.931-936, 2008.

TSE, G.; ALI, A.; PRASAD, S.K. et al. Atypical case of post-partum cardiomyopathy: an overlap syndrome with arrhythmogenic right ventricular cardiomyopathy. British heart journal, v. 1, 2015.

TSE, G.; YAN, B. P. Traditional and novel electrocardiographic conduction and repolarization markers of sudden cardiac death. Europace, v. 19, n.5, p. 1-10, 2016.

TURRINI, P.; ANGELINI, A.; THIENE, G. et al. Late potentials and ventricular arrhythmias in arrhythmogenic right ventricular cardiomyopathy. The American Journal of Cardiology, v. 83, n. 15, p. 1214-1219, 1999. 